\title{
Adsorption d'un colorant basique, Bleu de Méthylène, en solution aqueuse, sur un bioadsorbant issu de déchets agricoles de Cucumeropsis mannii Naudin
}

\author{
Kifline Milebudi KIFUANI " , Anatole KIFUANI KIA MAYEKO, Philippe NOKI \\ VESITULUTA, Bernard ILINGA LOPAKA, Gracien EKOKO BAKAMBO, \\ Blaise MBALA MAVINGA et Jérémie MUSWEMA LUNGUYA
}
Laboratoire de Chimie Organique Physique, Eau et Environnement (LACOPE), Département de Chimie et Industrie, Faculté des Sciences, Université de Kinshasa, B.P. 190, Kinshasa XI, RD Congo.
*Auteur correspondant, E-mail :kifuanikifline@gmail.com ; Tel :(00243) 895879602

\section{RESUME}

L'adsorption sur des bioadsorbants est un procédé courant pour éliminer les micropolluants de l'eau. Le but de notre travail est d'étudier l'adsorption du BM sur le BCM. L'adsorption du bleu de méthylène (BM) sur un bioadsorbant à base des téguments des graines de Cucumeropsis mannii Naudin (BCM) a été étudiée en réacteur discontinu. Les effets des paramètres ci-après ont été étudiés pour l'optimalisation de l'adsorption : masse de bioadsorbant, temps d'adsorption, concentration et $\mathrm{pH}$ de la solution de BM. La capacité ultime d'adsorption vraie a été évaluée à $89,41 \mathrm{mg} \mathrm{g}^{-1}$ à $\mathrm{pH} 6,67$, avec une surface spécifique de $251,87 \mathrm{~m}^{2} \mathrm{~g}^{-1}$. La capacité d'adsorption augmente avec la masse de bioadsorbant à cause de la disponibilité des sites libres. L'adsorption de BM sur BCM est meilleure en milieu basique, à cause des interactions électrostatiques entre la surface du BCM chargée négativement et les cations organiques de BM. Le BM est éliminé à 80,75\% dans les conditions optimales. Les isothermes obtenues sont toutes de type S. Le modèle de pseudo-ordre 1 est mieux approprié pour décrire l'adsorption de BM sur BCM, comparé au modèle de pseudo-ordre 2. L'adsorption de BM sur BCM est mieux décrite par le modèle de Langmuir. Le paramètre de séparation $\mathrm{R}_{\mathrm{L}}$ de Langmuir et le paramètre $1 / \mathrm{n}$ de Freundlich, inférieurs à 1 , indiquent que l'adsorption de BM sur BCM est favorable. Les résultats de cette étude montrent que le BCM est un bioadsorbant potentiel pour l'élimination des colorants organiques en solution aqueuse.

(C) 2018 International Formulae Group. All rights reserved.

Mots clés: Cucumeropsis mannii Naudin, bleu de méthylène, adsorption, bioadsorbant, isothermes, cinétiques, thermodynamiques.

\section{Adsorption of basic dye, Methylene Blue, in aqueous solution on bioadsorbent from agricultural waste of Cucumeropsis Naudin mannii}

\begin{abstract}
Adsorption on bioadsorbants is a common process for the removal of micropollutants from water. The purpose of our work was to study the adsorption of the MB on the BCM. The adsorption of methylene blue (MB) on a bioabsorbent prepared from Cucumeropsis Naudin mannii (BCM)'s seed teguments has been
\end{abstract}


studied in a batch reactor. The effects of some optimization parameters on the adsorption, such as the mass of absorbent, absorption time, concentration and $\mathrm{pH}$ of the solution of $\mathrm{MB}$, have been investigated. The ultimate adsorption capacity was found to be $89.41 \mathrm{mg} \mathrm{g}^{-1}$, at $\mathrm{pH} 6.67$, with a specific area of $251.87 \mathrm{~m}^{2} \mathrm{~g}^{-1}$. Adsorption capacity increases with the mass of the bioabsorbent, due to the availability of free sites. Adsorption of $\mathrm{MB}$ on $\mathrm{BCM}$ is higher in alkali medium because of electrostatic interactions between the negatively charged surfaces of BCM and organic cations of MB. When operational conditions are optimized, BM is removed up to $80.75 \%$ from the solution. All the experimental isotherms are S-shaped, and the pseudoorder 1 kinetic model seems to be more appropriate in this case as compared with the pseudo-order 2 model. The adsorption of BM on BCM is better described by the Langmuir model. The Langmuir $\mathrm{R}_{\mathrm{L}}$ separation parameter and the Freundlich 1/n parameter have both values under unity, indicated that the adsorption of MB on BCM is more favored. Result from this work show that BCM is a potential bioadsorbent for the removal of organic dyes in aqueous solution.

(C) 2018 International Formulae Group. All rights reserved.

Keywords: Cucumeropsis mannii Naudin, methylene blue, adsorption, bioadsorbent, isotherms, kinetics.

\section{INTRODUCTION}

Les colorants organiques sont couramment utilisés dans de nombreuses activités industrielles tels que les teintures du cuir, du papier, du textile, du bois, de la soie, des plastiques, ainsi que dans les industries cosmétiques, alimentaires et pharmaceutiques. Le bleu de méthylène est l'un des colorants le plus utilisé dans la teinture de plusieurs supports, notamment la soie, le coton et le bois et pour la coloration temporaire du papier. Il est utilisé comme indicateur coloré redox, colorant histologique, antiseptique en traitement des plaies superficielles (Atef, 2009). Par contact, le BM est susceptible de provoquer des brûlures oculaires chez l'homme ou l'animal. L'inhalation induit des difficultés respiratoires et l'ingestion buccale provoque des nausées, des vomissements, transpiration et sueurs froides abondantes (Atef, 2009 ; Kifuani, 2013).

Maurya et al. (2008), Vanessa et al. (2017) rapportent que les colorants organiques sont à la base de plusieurs effets sur la santé humaine ; certains colorants sont cancérigènes, mutagènes et tératogènes et sont susceptibles d'entrainer plusieurs affections comme les nausées, les hémorroïdes, les ulcères de la peau et de la muqueuse, des sévères irritations du système respiratoire. Madhura et al. (2014) indiquent que les eaux usées issues des procédés utilisant les colorants organiques présentent une DBO et DCO élevées, des matières solides élevées
Près de 5 à $25 \%$ des colorants appliqués sur les tissus ne sont pas fixés et se retrouvent dans les eaux usées et sont susceptibles de polluer les eaux, les espèces aquatiques notamment la faune et la flore. Les colorants organiques sont des polluants persistants. Leur élimination appelle des méthodes appropriées (Zidane et al., 2011). L'élimination des colorants organiques ainsi que celles d'autres polluants organiques ou inorganiques des eaux usées s'avère donc nécessaire. Plusieurs techniques d'élimination des colorants organiques des eaux usées sont utilisées et incluent des méthodes biologiques, chimiques et physiques. Ces méthodes englobent entre autres procédés la floculation, la précipitation, la filtration sur membrane, l'échange d'ions, l'ozonation, l'irradiation et l'adsorption (Kifuani et al., 2004 ; Hameed et al., 2007 ; Li et al., 2009; Muswema et al., 2010 ; Sun et al., 2012; Balogoun et al., 2015 ; Kassale et al., 2015 ; Berradi et al., 2016 ; Ousmaila et al., 2016 ; Oliveira et al., 2017). La plupart de ces méthodes sont coûteuses, exigent beaucoup d'énergie, produisent d'énormes quantités de boues ou génèrent des dérivés parfois plus toxiques que les produits initiaux eux-mêmes (Masson, 2015 ; Tchuifon, 2015).

Parmi les procédés de traitement des eaux polluées par les colorants organiques, l'adsorption s'avère être le plus efficace (Ertaş et al., 2010; Yao et Kouassi, 2015). Le charbon actif est l'un des adsorbants le plus utilisé à ce sujet (Sark et al., 2015). 
Cependant, le coût de production du charbon actif est assez souvent très élevé. Plusieurs travaux sont menés par de nombreux scientifiques du monde entier pour la préparation des matériaux adsorbants peux coûteux en utilisant des matériaux précurseurs peux coûteux, disponibles localement à partir des sources naturelles renouvelables, comme les déchets agro-industriels (Arivoli et al., 2009 ; Kazlauskieni et al., 2009 ; Li et al., 2009; Laximi et Ahmazzaman, 2010).

Nous nous sommes ainsi intéressés dans le présent travail à développer un bioadsorbant potentiel à l'adsorption des colorants organiques en utilisant comme matériaux précurseurs les déchets des téguments de Cucumeropsis mannii Naudin. Près de $40 \%$ des graines de courge sont constitués des téguments qui sont rejetés dans l'environnement comme déchets. Ils peuvent ainsi être à la base de la pollution de l'environnement. La conversion de ces téguments en bioadsorbant potentiel constitue ainsi un moyen de valorisation de déchets et d'assainissement de l'environnement. L'évaluation du potentiel adsorbant du bioadsorbant a été faite en utilisant le bleu de méthylène (BM) comme adsorbant.

\section{MATERIEL ET METHODES}

\section{Préparation et caractérisation du bioadsorbant}

Le bioadsorbant utilisé dans cette étude est préparé à partir des téguments de Cucumeropsis mannii Naudin. Les téguments sont lavés à l'eau de Robinet et ensuite avec de l'eau bidistillée. Les écorces lavées, broyées, tamisées (Tamis à mailles $\leq 1 \mathrm{~mm}$ ) sont séchées à la température du laboratoire $\left(29 \pm 1{ }^{\circ} \mathrm{C}\right)$ et après dans une étuve (DESPATCH Oven Co de type Elect) pendant 48 heures à $45{ }^{\circ} \mathrm{C}$. La poudre est alors conservée dans un container hermétique. La caractérisation préliminaire du Bioadsorbant Cucumeropsis mannii Naudin (BCM) ainsi obtenu a consisté en la détermination de l'humidité, de la matière sèche, des cendres, $\mathrm{du} \mathrm{pH}_{\mathrm{ZPC}}(\mathrm{pH}$ au point de zéro potentiel charge) et de la surface spécifique.

\section{Détermination de l'humidité}

L'humidité du bioadsorbant a été déterminée par gravimétrie de volatilisation. A cet effet, $5 \mathrm{~g}$ de bioadsorbant contenus dans un creuset sont placés dans une étuve (DESPATCH Oven Co de type Elect) à 105 ${ }^{\circ} \mathrm{C}$, pendant quelques jours jusqu'à poids constant. Après refroidissement dans un dessiccateur, la masse du bioadsorbant est ensuite déterminée. L'Humidité $(\% \mathrm{H})$ est alors calculée selon la relation :

$$
\% \mathrm{H}=\frac{\left(\mathrm{m}_{1}-\mathrm{m}_{2}\right) \cdot 100}{\mathrm{~m}_{1}}
$$

Avec, $\mathrm{m}_{1}=$ masse $\mathrm{du}$ bioadsorbant avant étuvage $(\mathrm{g})$ et $\mathrm{m}_{2}=$ masse du bioadsorbant après étuvage $(\mathrm{g})$.

\section{Détermination de la masse sèche}

La masse sèche est déterminée en déduisant la masse d'eau de la masse totale du bioadsorbant.

\section{Détermination des cendres}

Les cendres totales ont été déterminées par différence de la masse du bioadsorbant avant et après calcination dans un four à moufle (NABER, Modèle N7/H) à $800^{\circ} \mathrm{C}$.

\section{Détermination du $\mathrm{pH}_{Z P C}$ ( $\mathrm{pH}$ au point de zero potentiel charge)}

La détermination du $\mathrm{pH}_{\mathrm{ZPC}}$ est faite en recourant à la technique rapportée par Kifuani et al. (2012), Lopez-Ramon et al. (1999); c'est la «méthode de dérive de $\mathrm{pH} »(\mathrm{pH}$ drift method) et modifiée en tenant compte de la capacité d'adsorption du bioadsorbant. A cet effet, six solutions témoins de $\mathrm{NaCl} 0,01 \mathrm{~mol}$ $\mathrm{L}^{-1}$ de $\mathrm{pH}$ compris entre 2 et 12 sont préparées et on détermine avec exactitude leurs $\mathrm{pH}$. A $50 \mathrm{~mL}$ de chacune de ces solutions est ajouté $0,1 \mathrm{~g}$ de bioadsorbant. Les suspensions obtenues sont laissées sous agitation pendant 72 heures, à la température ambiante $(28 \pm 2$ ${ }^{\circ} \mathrm{C}$ ), et ensuite centrifugées (Centrifugeuse Labofuge 200 Heraeus). En pratique, le $\mathrm{pH}_{\mathrm{ZPC}}$ correspond au $\mathrm{pH}$ pour lequel la courbe $\mathrm{pH}_{\text {final }}=\mathrm{f}\left(\mathrm{pH}_{\text {initial }}\right)$ traverse la première bissectrice.

\section{Détermination de la surface spécifique}

La surface spécifique a été déterminée par la méthode d'adsorption de bleu de méthylène (MABM ou MBAM, methylene 
blue adsorption method, en anglais) qui consiste à étudier l'adsorption de bleu de méthylène, par une masse donnée du matériau adsorbant, par variation de volume de la solution de BM, jusqu'à une valeur maximale de la capacité d'adsorption. On obtient ainsi la capacité ultime d'adsorption vraie. La surface spécifique est calculée à partir de l'équation suivante (Kifuani, 2013) :

$\mathrm{S}_{\mathrm{BM}}=Q_{\mathrm{m}} \cdot N_{\mathrm{A}} \cdot s$

[2]

Avec, $S_{\mathrm{BM}}$ : surface spécifique déterminée en utilisant BM comme adsorbat $\left(\mathrm{m}^{2} \mathrm{~g}^{-1}\right) ; Q_{\mathrm{m}}$ : capacité maximale d'adsorption $\left(\mathrm{mg} \mathrm{g}^{-1}\right) ; s$ : aire occupée par une molécule de BM (175 $\left.\dot{\mathrm{A}}^{2}\right) ; N_{\mathrm{A}}$ : nombre d'Avogadro.

Les caractéristiques du bioadsorbant BCM déterminées sont présentées dans le Tableau 1.

\section{Préparation et analyse des solutions d'adsorbat}

Le bleu de méthylène a été choisi comme colorant organique modèle, à cause de sa large utilisation en laboratoire et comme colorant de teinture de la soie, du coton et du bois et aussi à cause de sa disponibilité et toxicité. C'est le prototype des colorants de taille moyenne. La solution mère de BM 0,737 $\mathrm{g} \mathrm{L}^{-1}$ est préparée par dissolution de la masse requise de BM dans $2 \mathrm{~L}$ de solution, sans purification préalable. Les solutions filles sont préparées par dilutions successives de la solution mère pour obtenir $1 \mathrm{~L}$ des solutions variant de $1 \mathrm{mg} \mathrm{L}^{-1}$ à $100 \mathrm{mg} \mathrm{L}^{-1}$. Les solutions de $\mathrm{BM}$ avant et après adsorption sont analysées par spectrophotométrie UVVis (Spectrophotomètre HACK, modèle 1115) à la longueur d'onde déterminée expérimentalement par balayage du domaine de longueurs d'onde allant de $400 \mathrm{~nm}$ à 700 $\mathrm{nm}$, pour les différents $\mathrm{pH}$ étudiés. Les concentrations sont alors déduites à partir de la loi de Beer-Lambert $\boldsymbol{A}=\boldsymbol{\varepsilon} \boldsymbol{\ell C}$ [3]

Avec: $A$ : absorbance de la solution ; $\varepsilon$, coefficient d'absorption molaire de la substance $\left(\mathrm{L} \mathrm{mg}^{-1} \mathrm{~cm}^{-1}\right) ; \ell$, longueur $\mathrm{du}$ chemin optique (cm) et $C$, la concentration de la substance $\left(\mathrm{mg} \mathrm{L}^{-1}\right)$. L'étalonnage est réalisé pour les concentrations de 1 à $100 \mathrm{mg}$
$\mathrm{L}^{-1}$. La Figure 1 donne la structure du bleu de méthylène.

\section{Essais d'adsorption}

\section{Effet de la masse de bioasorbant}

Les essais d'adsorption en fonction de la masse de Bioadsorbant ont été effectués en régime discontinu dans des adsorbeurs hermétiques (LACOPE ADS X-3), avec les masses de $10 \mathrm{mg}$ à $1000 \mathrm{mg}$ de Bioadsorbant. La solution de BM $50 \mathrm{mg} \mathrm{L^{- }}$ ${ }^{1}$ est utilisée pour tous les essais d'adsorption en fonction de la masse de Bioadsorbant. L'adsorption en fonction de la masse de bioadsorbant a été étudiée en solution aqueuse au $\mathrm{pH}$ d'autoéquilibre $(6,67)$. Pour chaque essai d'adsorption, une masse de bioadsorbant est placée dans l'adsorbeur; on ajoute $100 \mathrm{~mL}$ de la solution de BM. La suspension est agitée pendant 350 minutes, temps situé dans le domaine de l'équilibre pour toutes les masses utilisées. Après agitation, la suspension est centrifugée (Centrifugeuse LABOFUGE 200 HERAEUS) pendant 30 minutes à 3000 rpm ; le centrifugeât est alors analysé à la longueur d'onde requise. La capacité d'adsorption du bioadsorbant BCM a été calculée selon la formule :

$$
\boldsymbol{Q}_{e}=\frac{\left(\mathrm{C}_{\mathrm{o}}-\mathrm{C}_{\mathrm{e}}\right) \mathrm{V}}{\mathrm{m}_{\mathrm{B}}}
$$

Avec $Q_{e}$, la capacité d'adsorption du bioadsorbant, $C_{0}$, la concentration initiale (mg/L), $V$, le volume de la solution de BM $(\mathrm{L}), C_{\mathrm{e}}$, la concentration à l'équilibre $(\mathrm{mg} / \mathrm{L})$, et $\quad m_{\mathrm{B}}$, la masse du bioadsorbant utilisée.

Le pourcentage d'adsorption (\% Ads) du BM sur le bioadsorbant BCM est donné par la relation suivante :

$$
\% \operatorname{Ads}=\frac{\mathrm{C}_{\mathrm{o}}-\mathrm{C}_{\mathrm{e}}}{\mathrm{C}_{\mathrm{o}}} \times 100
$$

\section{Effet du temps d'adsorption}

Les essais d'adsorption en fonction du temps d'adsorption ont été effectués selon le même protocole expérimental que pour les essais d'adsorption en fonction de la masse de bioadsorbant. Les masses de $10 \mathrm{mg}$ à 1000 mg de Bioadsorbant ont été utilisées et le 
temps d'agitation a été varié de 0 à 450 minutes.

\section{Effet du pH de la solution}

L'effet du pH de la solution de BM sur la capacité d'adsorption du Bioadsorbant a été étudié aux pH suivants : 3, 4, 5, 8, 10 et 12 . Les essais sont effectués en régime discontinu en utilisant $100 \mathrm{~mL}$ de la solution de BM 50 $\mathrm{mg} \mathrm{L^{-1 }}$ et $800 \mathrm{mg}$ de Bioadsorbant, masse optimale déterminée expérimentalement en étudiant l'adsorption en fonction de la masse de bioadsorbant. Le temps d'adsorption est varié de 0 à 450 minutes. Le même protocole expérimental utilisé pour les essais d'adsorption en fonction de la masse a été appliqué.

\section{Effet de la concentration initiale}

L'effet de la concentration initiale est étudié en régime discontinu pour les concentrations des solutions de BM de $1 \mathrm{mg}$ $\mathrm{L}^{-1}$ à $100 \mathrm{mg} \mathrm{L}^{-1}$ en vue de déterminer les isothermes d'adsorption. Le temps d'agitation est fixé à 180 minutes, temps se situant dans le domaine d'équilibre, pour tous les $\mathrm{pH}$ étudiés. Le protocole expérimental est le même que celui suivi pour l'adsorption en fonction de la masse du bioadsorbant, sauf qu'ici tous les essais sont effectués en même temps.

\section{Modélisation de la cinétique d'adsorption}

La modélisation de la cinétique d'adsorption de BM sur le Bioadsorbant $\mathrm{BCM}$ est faite à l'aide des modèles cinétiques de la réaction de surface. Les modèles cinétiques couramment utilisés pour la réaction de surface sont ceux de pseudo-ordre 1 et de pseudo-ordre 2. Les équations habituellement utilisées sont les suivantes :

Modèle de pseudo-ordre 1 ou modèle de Lagergren :

$$
\begin{aligned}
& \frac{\mathrm{dq}_{\mathrm{t}}}{\mathrm{dt}}=\mathrm{k}_{1}\left(\mathrm{q}_{\mathrm{e}}-\mathrm{q}_{\mathrm{t}}\right) \\
& \ln \left(\mathrm{q}_{\mathrm{e}}-\mathrm{q}_{\mathrm{t}}\right)=\ln \mathrm{q}_{\mathrm{e}}-\mathrm{k}_{1} \mathrm{t}
\end{aligned}
$$

Modèle de pseudo-ordre 2

$$
\frac{\mathrm{dq}_{\mathrm{t}}}{\mathrm{dt}}=\mathrm{k}_{2}\left(\mathrm{q}_{\mathrm{e}}-\mathrm{q}_{\mathrm{t}}\right)^{2}
$$

$$
\frac{1}{\left(q_{e}-q_{t}\right)}=\frac{1}{q_{e}}+k_{2} t
$$

Dans cette étude, nous utilisons les équations linéaires de pseudo-ordre 1 et de pseudo-ordre 2 pour l'adsorption, développées par Kifuani et al. (2012).

Equation de Kifuani pour la réaction cinétique de pseudo-ordre 1 :

$$
\ln \frac{\mathrm{q}_{\mathrm{e}}}{\left(\mathrm{q}_{\mathrm{e}}-\mathrm{q}_{\mathrm{t}}\right)}=\mathrm{k}_{1} \mathrm{t} \text { [10] }
$$

$q_{\mathrm{e}}$ : concentration maximale de soluté A adsorbée, quand l'équilibre est atteint (correspond à la capacité maximale d'adsorption, $q_{\mathrm{m}}\left(\mathrm{mg} \mathrm{g}^{-1}\right) ; q_{\mathrm{t}}$ : concentration de A adsorbée au temps $t$, et correspondant à la capacité apparente d'adsorption, $q\left(\mathrm{mg} \mathrm{g}^{-1}\right)$; $k_{1}$, constante de vitesse de la réaction de pseudo-ordre $1\left(\mathrm{~min}^{-1}\right)$.

En portant $\ln \frac{\mathrm{q}_{\mathrm{e}}}{\left(\mathrm{q}_{\mathrm{e}}-\mathrm{q}_{\mathrm{t}}\right)}$ en fonction du temps, $t$, on obtient une droite dont la pente correspond à $k_{1}$, la constante de vitesse de la réaction d'adsorption de surface de pseudoordre 1.

Equation de Kifuani pour la réaction cinétique de pseudo-ordre 2 :

$$
\frac{\mathrm{q}_{\mathrm{t}}}{\mathrm{q}_{\mathrm{e}}\left(\mathrm{q}_{\mathrm{e}}-\mathrm{q}_{\mathrm{t}}\right)}=\mathrm{k}_{2} \mathrm{t}
$$

Avec, $k_{2}$ : constante de vitesse de la réaction de pseudo-ordre $2\left(\mathrm{~g} \mathrm{mg}^{-1} \mathrm{~min}^{-1}\right)$.

Lorsqu'on porte $\frac{q_{t}}{q_{e}\left(q_{e}-q_{t}\right)}$ en fonction de $t$, on obtient une droite dont la pente correspond à $k_{2}$, la constante de vitesse de la réaction d'adsorption de surface de pseudoordre 2 .

\section{Isothermes d'adsorption}

Les isothermes d'adsorption ont été obtenues en étudiant l'adsorption de BM sur le Bioadsorbant BCM. $100 \mathrm{~mL}$ des solutions de concentrations $1 \mathrm{mg} \mathrm{L}^{-1}$ à $100 \mathrm{mg} \mathrm{L}^{-1}$ ont été utilisés. Les essais ont été effectués avec 
$800 \mathrm{mg}$ de bioadsorbant, masse optimale déterminée expérimentalement.

\section{Modélisation des isothermes d'adsorption}

La modélisation des isothermes d'adsorption a été faite en recourant aux modèles d'équilibre de Langmuir et de Freundlich (Kifuani, 2013).

\section{Le modèle de Langmuir}

Le modèle de Langmuir a été utilisé sous sa forme linéaire donnée par l'équation ci-dessous :

$$
\frac{1}{\mathrm{Q}_{\mathrm{e}}}=\frac{1}{\mathrm{Q}_{\mathrm{m}}}+\frac{1}{\mathrm{Q}_{\mathrm{m}} \mathrm{K}_{\mathrm{L}}} \cdot \frac{1}{\mathrm{C}_{\mathrm{e}}}
$$

Avec : $Q_{\mathrm{e}}$ : quantité du soluté fixée par unité de masse de l'adsorbant (capacité apparente d'adsorption); $Q_{\mathrm{m}}$ : quantité maximale du soluté fixée par unité de masse d'adsorbant. En portant $1 / Q_{\mathrm{e}}$ en fonction de $1 / C_{\mathrm{e}}$, cette équation permet de calculer les paramètres $Q_{\mathrm{m}}$ et $K_{\mathrm{L}}$, à partir de l'ordonnée à l'origine et de la pente, respectivement.

\section{Le modèle de Freundlich}

L'équation linéaire de Freundlich a été utilisée:

$$
\log Q_{\mathrm{e}}=\log K_{\mathrm{F}}+\frac{1}{\mathrm{n}} \log C_{\mathrm{e}}
$$

$Q_{\mathrm{e}}$ et $C_{\mathrm{e}}$ sont les concentrations du soluté dans le complexe solide et en solution, respectivement; $K_{\mathrm{F}}$ constante de Freundlich, une constante qui est relative à la capacité d'adsorption; c'est donc la capacité d'adsorption lorsque la concentration à l'équilibre est unitaire.

La constante adimensionnelle $\mathrm{n}$ est en rapport avec l'intensité d'adsorption. En portant $\log Q_{\mathrm{e}}$ en fonction de $\log C_{\mathrm{e}}$, cette équation permet de calculer les paramètres $K_{\mathrm{L}}$ et $1 / \mathrm{n}$ à partir de l'ordonnée à l'origine et de la pente respectivement.

Tableau 1: Caractéristiques du Bioadsorbant BCM.

\begin{tabular}{lc}
\hline \multicolumn{1}{c}{ Paramètres } & Valeurs \\
\hline Granulométrie & $\leq 1 \mathrm{~mm}$ \\
Humidité (\%) & 7,08 \\
Matières sèches (\%) & 92,92 \\
Cendres (\%) & 03,20 \\
$\mathrm{pH}_{\mathrm{ZPC}}$ & 05,02 \\
$\mathrm{~S}_{\mathrm{BM}}\left(\mathrm{m}^{2} \mathrm{~g}^{-1}\right)$ & 251,87 \\
\hline
\end{tabular}<smiles></smiles>

Figure 1: Structure du bleu de méthylène. 


\section{RESULTATS}

L'adsorption du bleu de méthylène a été étudiée en fonction de la masse de bioadsorbant, du temps d'adsorption, du $\mathrm{pH}$ de la solution de BM et de la concentration initiale de la solution de BM. Pour chaque paramètre, la capacité ultime d'adsorption apparente et le pourcentage d'adsorption ont été calculés. Les résultats obtenus sont présentés ci-après.

\section{Effet de la masse du Bioadsorbant}

Pour déterminer la masse optimale nécessaire pour éliminer le $\mathrm{BM}, 100 \mathrm{~mL}$ de la solution de BM $50 \mathrm{mg} \mathrm{L}^{-1}$ sont mis en contact avec différentes masses de Bioadsorbant. Les résultats présentés par la Figure 2 montrent un accroissement du pourcentage d'adsorption avec la masse du Bioadsorbant. Le pourcentage d'adsorption passe ainsi de $24,25 \%$ à $80,63 \%$, lorsque la masse du Bioadsorbant passe de 10 à $400 \mathrm{mg}$ (Tableau 1). A partir de $800 \mathrm{mg}$, le pourcentage d'adsorption demeure pratiquement constante jusqu'à $1000 \mathrm{mg}$. La Figure 3, par contre, montre une diminution de la capacité ultime d'adsorption $\left(Q_{\mathrm{m}}\right)$ lorsque la masse du Bioadsorbant augmente jusqu'à une valeur constante, à partir de $800 \mathrm{mg}$ de BCM. Ces résultats montrent que la capacité ultime d'adsorption diminue brutalement avec l'accroissement de la masse de Bioadsorbant de $10 \mathrm{mg}$ à $200 \mathrm{mg}$ et ensuite légèrement jusqu'à la masse de $800 \mathrm{mg}$, masse à partir de laquelle elle devient pratiquement constante.

\section{Effet du temps d'adsorption}

L'effet du temps sur l'adsorption a été étudié en mettant en contact une solution de BM $50 \mathrm{mg} \mathrm{L}^{-1}$ avec une masse donnée de Bioadsorbant. Les résultats obtenus consignés dans la Figure 4 indiquent une diminution progressive de la concentration réduite avec le temps de contact dépendant de la masse de bioadsorbant; et après un temps donné, la concentration réduite demeure constante. Le pourcentage d'adsorption de BM sur BCM augmente avec le temps de contact. Au début, le pourcentage d'adsorption augmente faiblement jusqu'à 200 minutes, quand on utilise $10 \mathrm{mg}$ à $400 \mathrm{mg}$ de Bioadsorbant ou rapidement jusqu'à moins de 10 minutes, pour les masses de $800 \mathrm{mg}$ et $1000 \mathrm{mg}$ (Figure 5).
La capacité d'adsorption apparente $(Q)$ augmente aussi avec le temps de contact BCM-BM jusqu'à atteindre une valeur maximale qui reste constante, malgré l'accroissement du temps (Figure 6). Cette valeur maximale correspond à la capacité ultime d'adsorption. Le temps d'équilibre d'adsorption est dépendant de la masse du bioadsorbant utilisée. Ce temps est plus court pour les masses élevées de Bioadsorbant. Les résultats consignés dans le Tableau 2 montrent que le temps d'équilibre passe de 330 minutes à 60 minutes, quand la masse augmente de 10 à 1000 mg, respectivement.

\section{Effet du pH sur le pourcentage d'adsorption et la capacité d'adsorption}

L'effet du $\mathrm{pH}$ sur l'adsorption a été étudié avec des solutions de BM $50 \mathrm{mg} \mathrm{L}^{-1}$ pour les $\mathrm{pH} 3,4,5,8,10$ et 12 , en utilisant une masse de Bioadsorbant de $800 \mathrm{mg}$, masse optimale déterminée expérimentalement. Les courbes présentées dans la Figure 7 montrent une diminution de la concentration réduite en fonction du temps d'adsorption, à différents $\mathrm{pH}$, jusqu'à une valeur minimale qui reste constante à partir de 150 minutes, dans la plupart des cas. Pour tous les pH étudiés, on observe une augmentation du pourcentage d'adsorption et de la capacité d'adsorption en fonction du temps de contact BCM-BM, jusqu'atteindre une valeur limite qui ne change plus malgré l'accroissement du temps (Figures 8 et 9).

Les courbes des Figures 10 et 11 ainsi que les résultats résumés dans le Tableau 3 montrent une augmentation du pourcentage d'adsorption de BM sur le bioadsorbant BCM avec le $\mathrm{pH}$ de la solution de $\mathrm{BM}$, jusqu'à une valeur pratiquement constante à partir du $\mathrm{pH}$ 10.

\section{Modélisation de l'adsorption}

Les paramètres cinétiques de la réaction de surface de pseudo-ordre 1 et pseudo-ordre 2 sont donnés dans le Tableau 4. Les isothermes obtenues sont reprises sur la Figure 12. Les paramètres de Langmuir et Freundlich calculés sont consignés dans le Tableau 5. Les courbes de linéarisation ne sont pas reprises ici. 


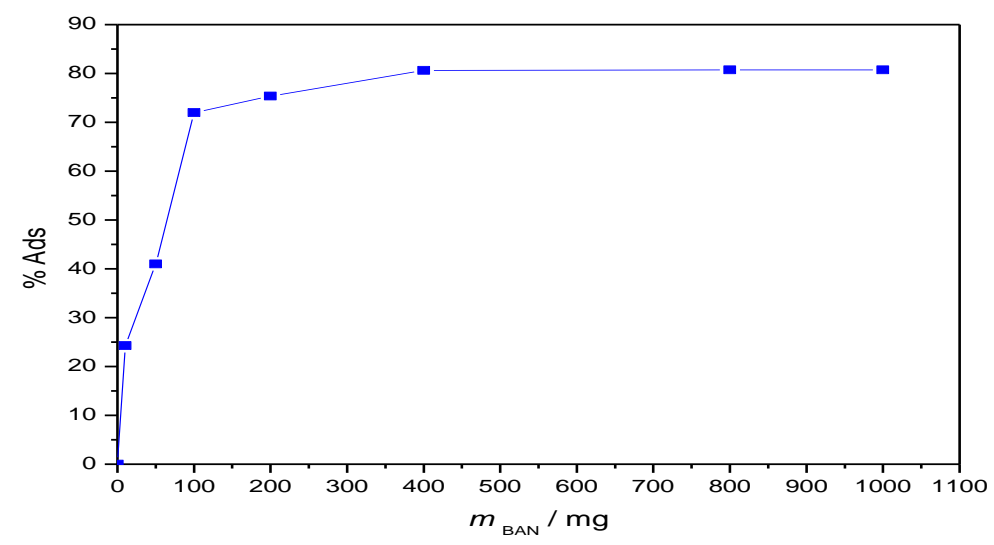

Figure 2 : Evolution de $Q_{\mathrm{m}}$ en fonction de la masse de BA. $V=100 \mathrm{~mL} ; C_{\mathrm{i}}=50,0 \pm 0,4 \mathrm{mg} \mathrm{L}^{-1}$; $\mathrm{pH}=6,67 \pm 0,01 ; T=29 \pm 1{ }^{\circ} \mathrm{C}$.

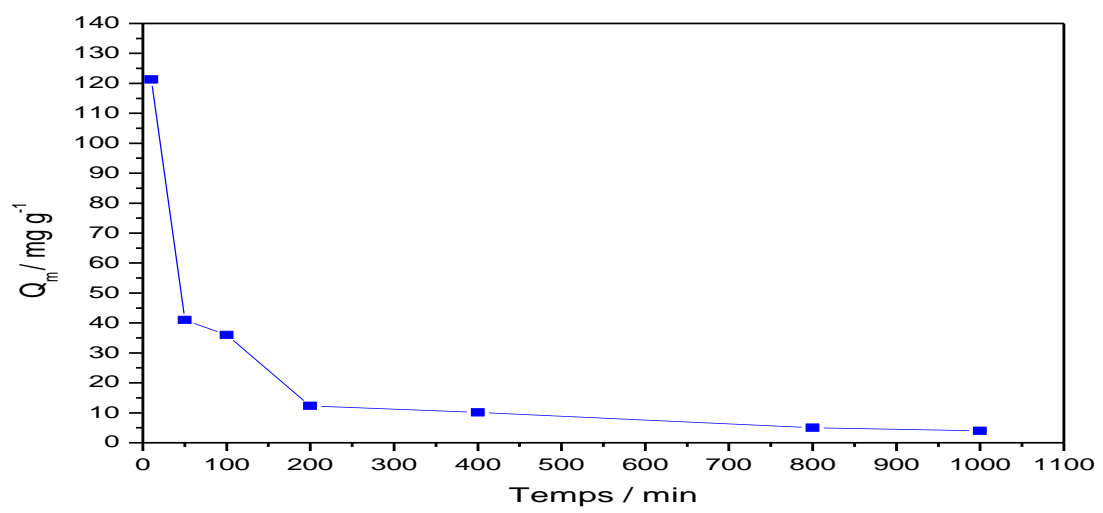

Figure 3 : Evolution de la Capacité d'adsorption en fonction de la masse du Bioadsorbant. $V=100$ $\mathrm{mL} ; C_{\mathrm{i}}=50,0 \pm 0,4 \mathrm{mg} \mathrm{L}^{-1} ; \mathrm{pH}=6,67 \pm 0,01 ; T=29 \pm 1^{\circ} \mathrm{C}$.

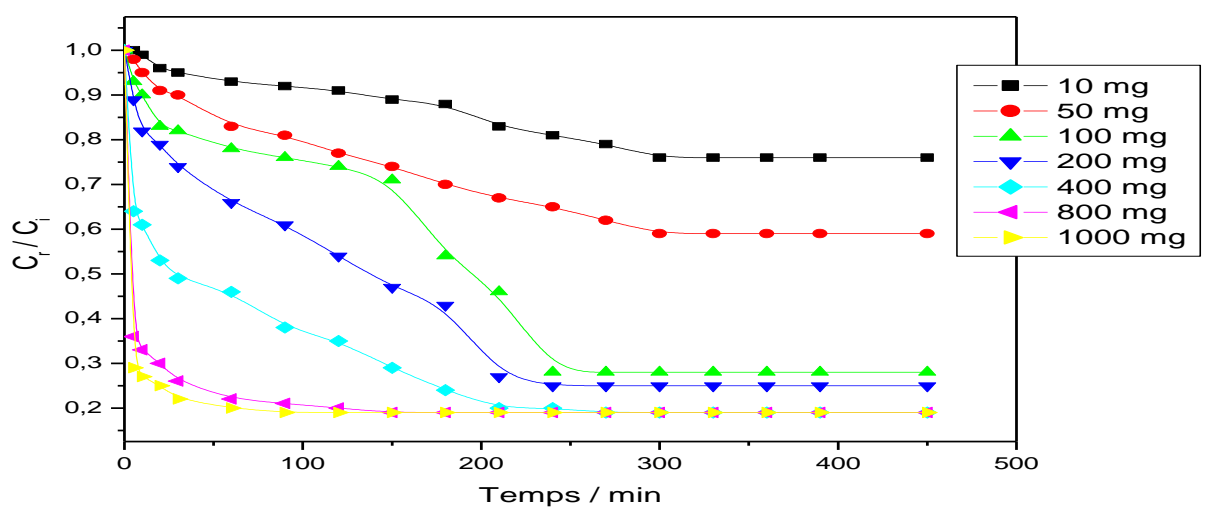

Figure 4 : Evolution de la concentration réduite de $\mathrm{BM}$ en fonction du temps pour différentes masses de Bioadsorbant. $V=100 \mathrm{~mL} ; C_{\mathrm{i}}=50,0 \pm 0,4 \mathrm{mg} \mathrm{L}^{-1} ; \mathrm{pH}=6,67 \pm 0,01 ; T=29 \pm 1{ }^{\circ} \mathrm{C}$. 


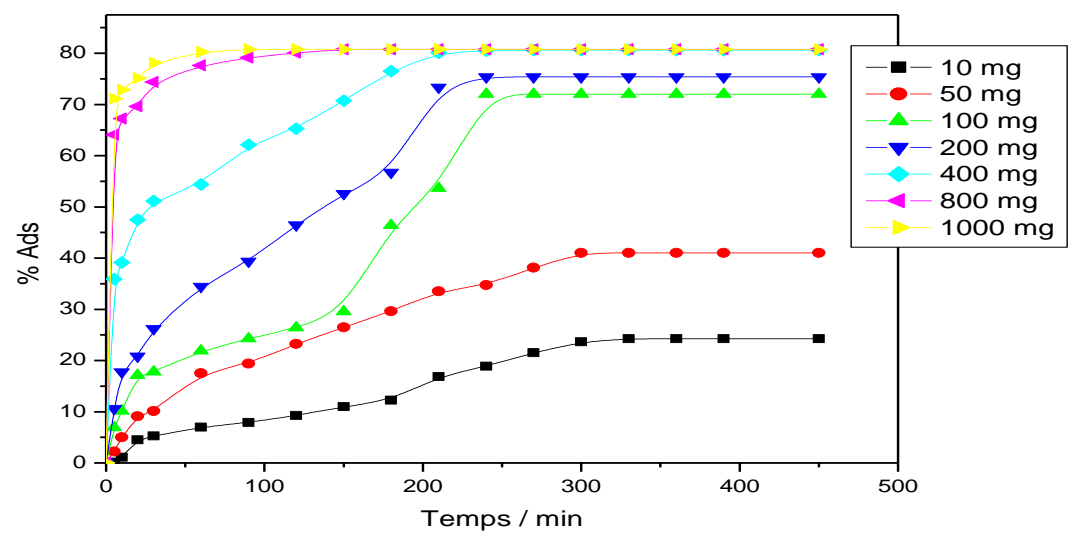

Figure 5: Evolution du pourcentage d'adsorption en fonction du temps pour différentes masses du bioadsorbant. $V=100 \mathrm{~mL} ; C_{\mathrm{i}}=50,0 \pm 0,4 \mathrm{mg} \mathrm{L}^{-1} ; \mathrm{pH}=6,67 \pm 0,01 ; T=29 \pm 1{ }^{\circ} \mathrm{C}$.

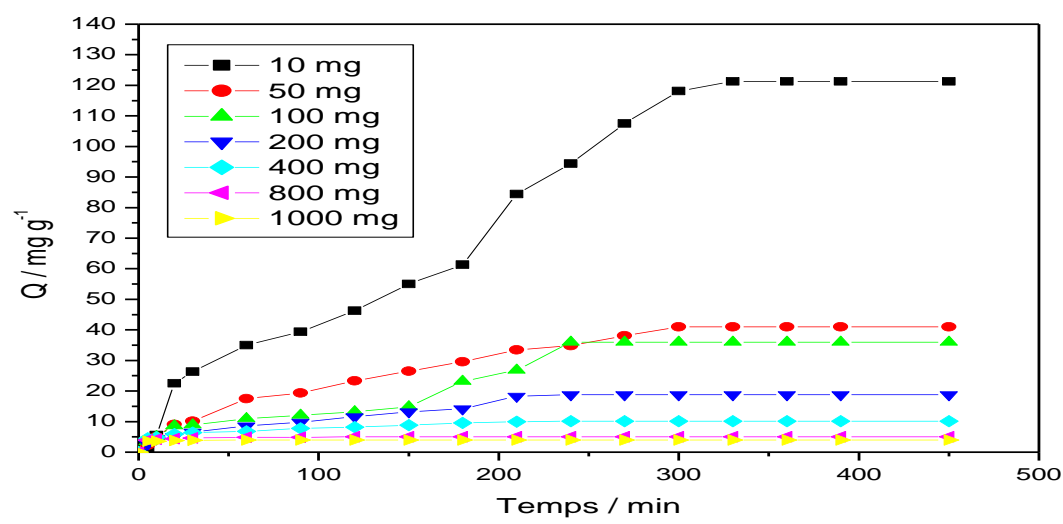

Figure 6: Evolution de la capacité d'adsorption du Bioadsorbant en fonction du temps pour différentes masses de Bioadsorbant. $V=100 \mathrm{~mL} ; C_{\mathrm{i}}=50,0 \pm 0,4 \mathrm{mg} \mathrm{L}^{-1} ; \mathrm{pH}=6,67 \pm 0,01 ; T=29 \pm 1{ }^{\circ} \mathrm{C}$.

Tableau 2: Capacités ultimes d'adsorption, pourcentage d'adsorption et temps d'équilibre d'adsorption pour différentes masses de Bioadsorbant BCM. $V=100 \mathrm{~mL} ; \mathrm{pH}=6,67 ; T=29 \pm 1^{\circ} \mathrm{C}$.

\begin{tabular}{lccc}
\hline $\boldsymbol{m}_{\mathbf{B C M}}(\mathbf{g})$ & $\boldsymbol{Q}_{\mathbf{m}}\left(\mathbf{m g ~ g}^{-\mathbf{1}}\right)$ & \% Ads & TE( $\mathbf{~ i n})$ \\
\hline 10 & 101,3 & 24,25 & 330 \\
50 & 41,0 & 41,0 & 300 \\
100 & 36,0 & 72,0 & 240 \\
200 & 18,8 & 75,38 & 210 \\
400 & 10,1 & 80,63 & 180 \\
800 & 5,0 & 80,75 & 150 \\
1000 & 4,0 & 80,75 & 60 \\
\hline
\end{tabular}

TE, temps d'équilibre 


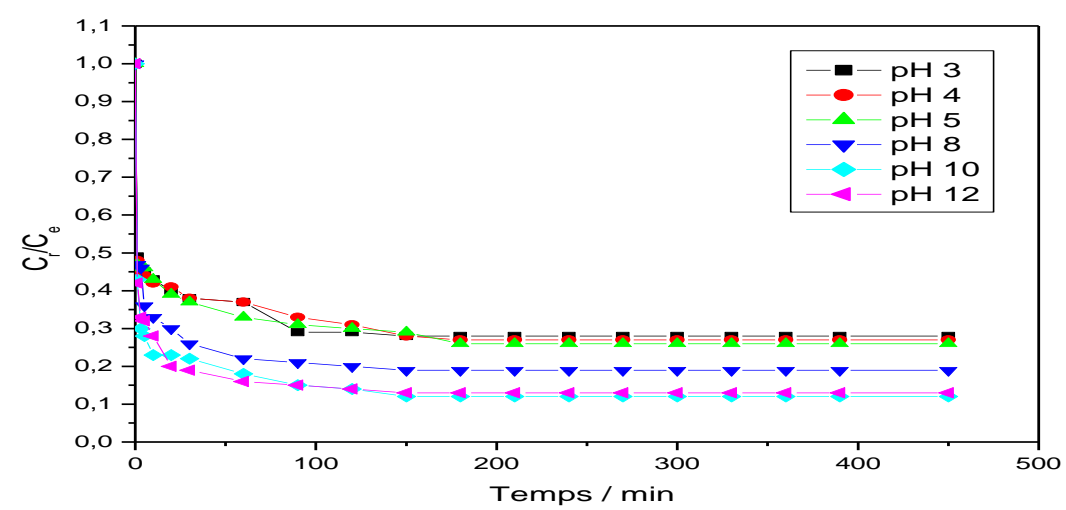

Figure 7 : Evolution de la concentration réduite en fonction du temps àdifférents $\mathrm{pH} . \quad V=100 \mathrm{~mL}$; $C_{\mathrm{i}}=50,0 \pm 0,4 \mathrm{mg} \mathrm{L}^{-1} ; m_{\mathrm{BA}}=800 \mathrm{mg} ; T=29 \pm 1^{\circ} \mathrm{C}$.

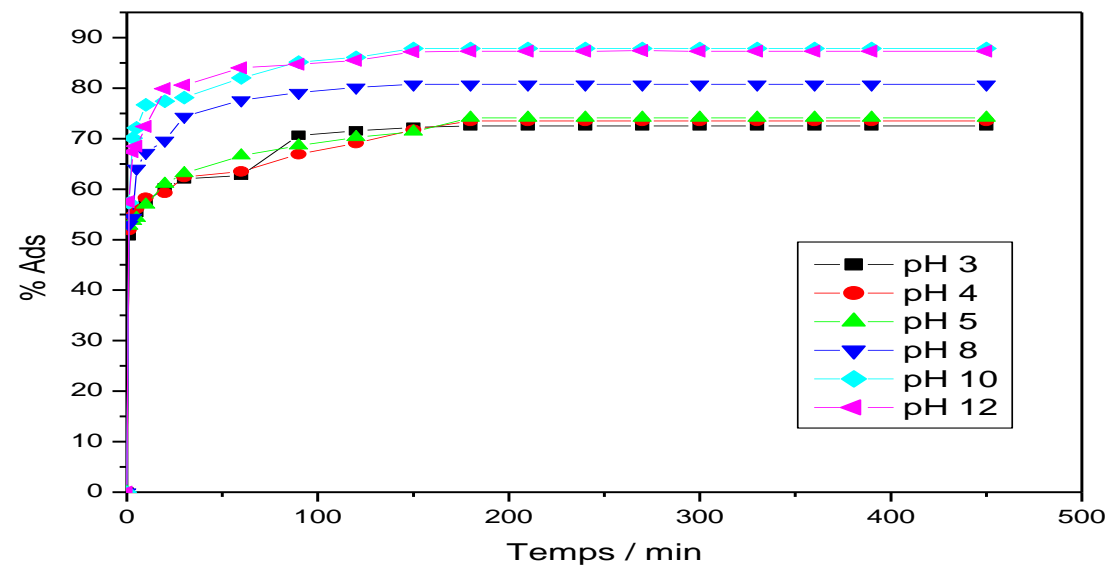

Figure 8 : Evolution du pourcentage d'adsorption en fonction du temps à différents $\mathrm{pH} . V=100 \mathrm{~mL}$; $C_{\mathrm{i}}=50,0 \pm 0,4 \mathrm{mg} \mathrm{L}^{-1} ; m_{\mathrm{BA}}=800 \mathrm{mg} ; T=29 \pm 1^{\circ} \mathrm{C}$.

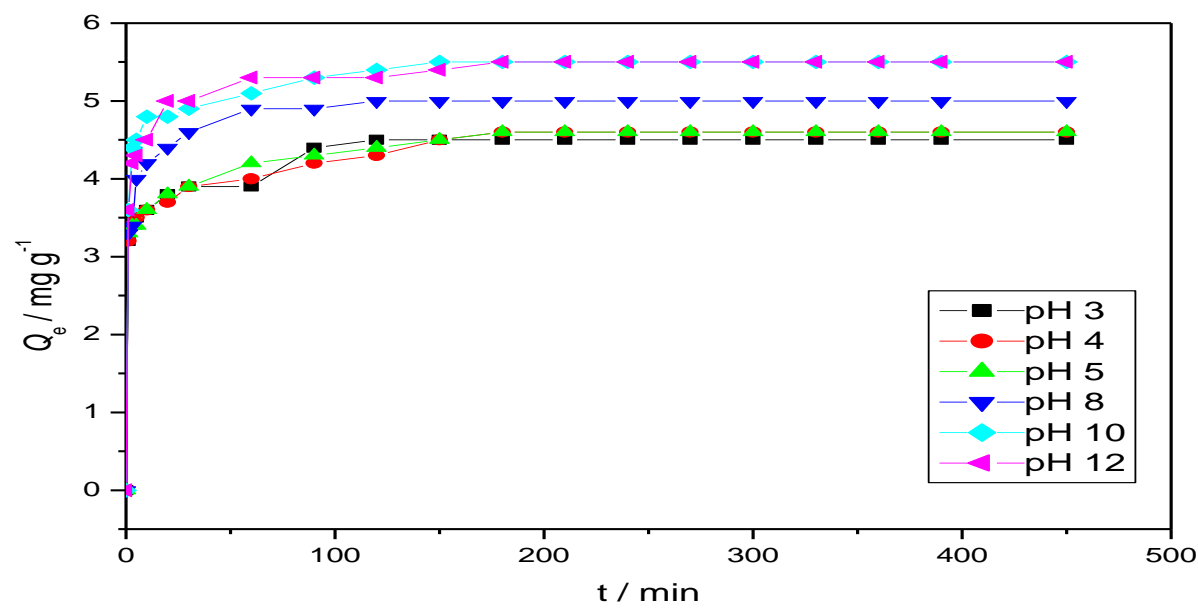

Figure 9 : Evolution de la capacité d'adsorption en fonction du temps à différents $\mathrm{pH} . V=100 \mathrm{~mL}$; $C_{\mathrm{i}}=50,0 \pm 0,4 \mathrm{mg} \mathrm{L}^{-1} ; m_{\mathrm{BA}}=800 \mathrm{mg} ; T=29 \pm 1^{\circ} \mathrm{C}$. 


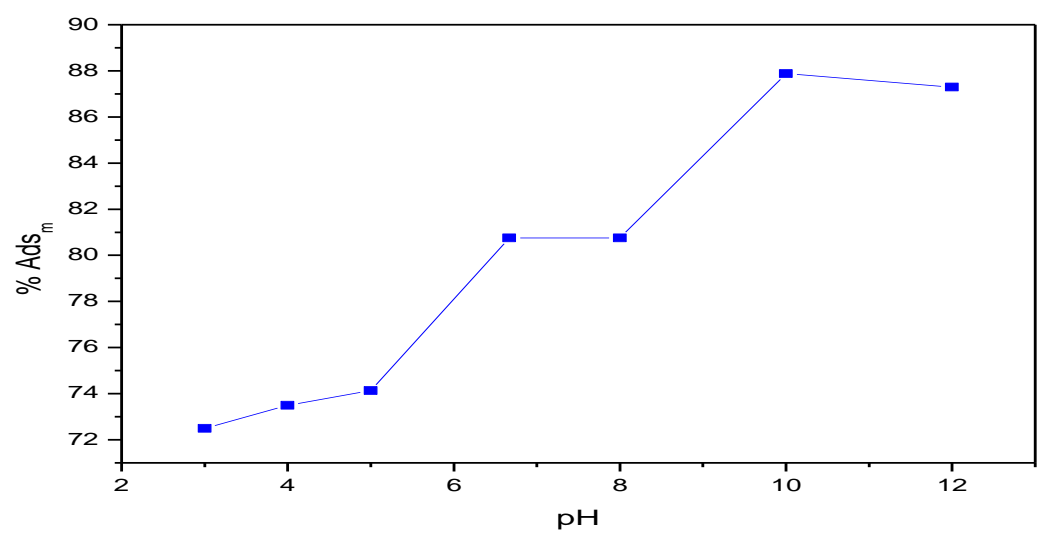

Figure 10: Evolution du \% Ads en fonction du pH. $V=100 \mathrm{~mL} ; C_{\mathrm{i}}=50,0 \pm 0,4 \mathrm{mg} \mathrm{L}^{-1} ; m_{\mathrm{BA}}=800 \mathrm{mg} ; T=29 \pm 1$ ${ }^{\circ} \mathrm{C}$.

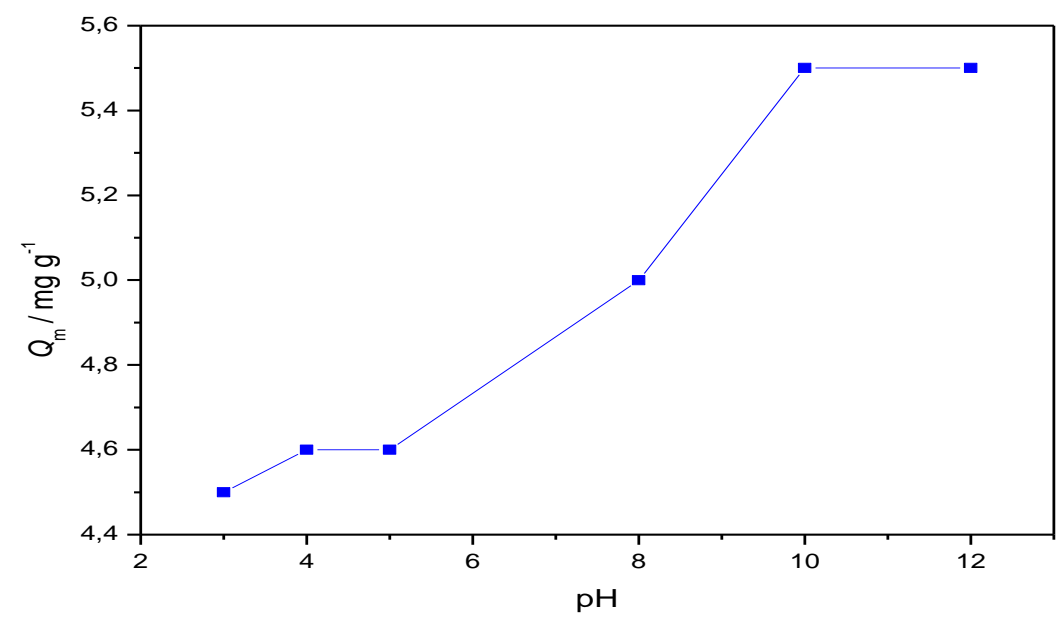

Figure 11 : Evolution de la Capacité d'adsorption en fonction du pH. $V=100 \mathrm{~mL} ; C_{\mathrm{i}}=50,0 \pm 0,4 \mathrm{mg} \mathrm{L}^{-1}$; $m_{\mathrm{BA}}=800 \mathrm{mg} ; T=29 \pm 1{ }^{\circ} \mathrm{C}$.

Tableau 3: Capacités ultimes d'adsorption, pourcentage d'adsorption et temps d'équilibre d'adsorption pour différents $\mathrm{pH}$ des solutions de BM.

\begin{tabular}{cccc}
\hline $\boldsymbol{m}_{\mathbf{B A}}(\mathbf{g})$ & $\boldsymbol{Q}_{\mathbf{m}}\left(\mathbf{m g ~ g}^{-\mathbf{1}}\right)$ & \% Ads & TE ( min) \\
\hline 3 & 5,5 & 72,50 & 150 \\
4 & 4,6 & 73,50 & 180 \\
5 & 4,6 & 74,13 & 180 \\
8 & 5,0 & 80,75 & 150 \\
10 & 5,5 & 87,88 & 150 \\
12 & 5,5 & 87,29 & 180 \\
\hline
\end{tabular}

Note : TE, temps d'équilibre ; $m_{\mathrm{BCM}}=800 \mathrm{mg} ; V=100 \mathrm{~mL} ; T=29 \pm 1{ }^{\circ} \mathrm{C}$. 
Tableau 4 : Paramètres de pseudo-ordre 1 et de pseudo-ordre 2 pour l'adsorption de BM sur BCM.

\begin{tabular}{lcccc}
\hline $\mathrm{pH}$ & \multicolumn{2}{c}{ Paramètres de pseudo-ordre 1 } & \multicolumn{2}{c}{ Paramètres de pseudo-ordre 2 } \\
\cline { 2 - 5 } & $\boldsymbol{k}_{\mathbf{1}}\left(\mathbf{m i n}^{-\mathbf{1}}\right)$ & $\mathbf{R}^{\mathbf{2}}$ & $\boldsymbol{k}_{\mathbf{2}}\left(\mathbf{g ~ m}^{-\mathbf{1}} \mathbf{~ m i n}^{-\mathbf{1}}\right)$ & $\mathbf{R}^{\mathbf{2}}$ \\
\hline 3 & 0,0231 & 0,8468 & 0,0074 & 0,8893 \\
4 & 0,0146 & 0,9464 & 0,0052 & 0,9538 \\
5 & 0,0162 & 0,9893 & 0,0056 & 0,9286 \\
8 & 0,0334 & 0,9126 & 0,0111 & 0,7865 \\
10 & 0,0204 & 0,9397 & 0,0073 & 0,7809 \\
12 & 0,9243 & 0,8543 & 0,0064 & 0,7004 \\
\hline
\end{tabular}

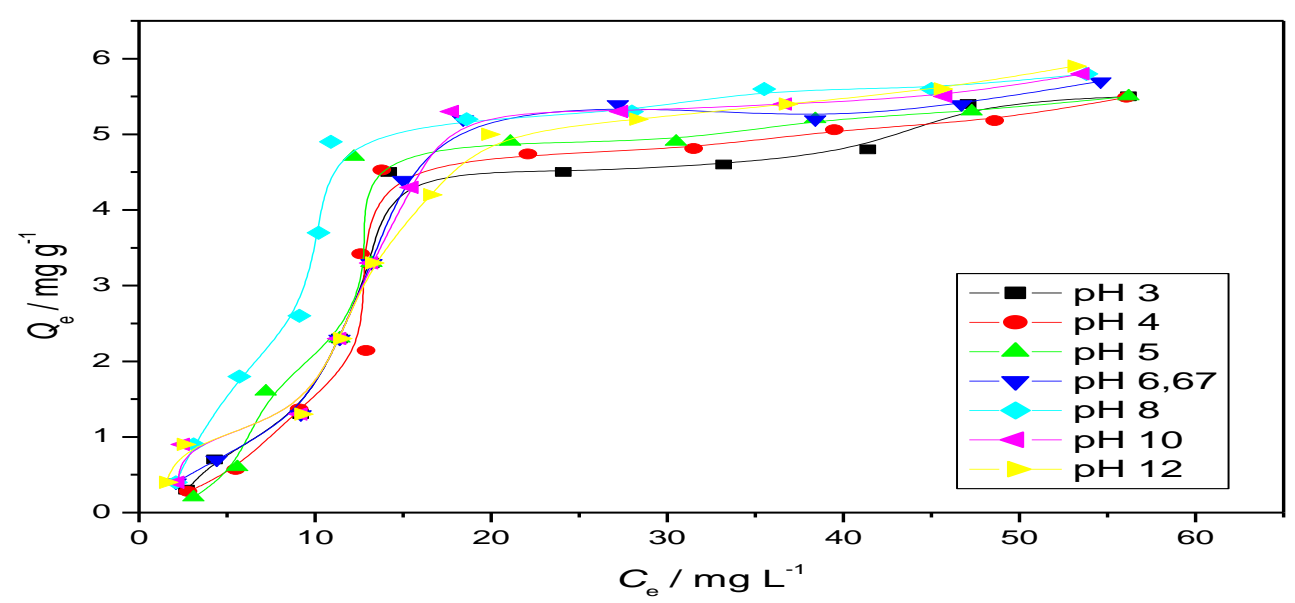

Figure 12 : Les isothermes d'adsorption de BM sur Bioadsorbant à différents pH. $V=100 \mathrm{~mL} ; m_{\mathrm{BA}}=$ $800 \mathrm{mg} ; T=29 \pm 1{ }^{\circ} \mathrm{C}$.

Tableau 5: Paramètres de Langmuir et de Freundlich pour l'adsorption de BM sur BCM.

\begin{tabular}{cccccccc}
\hline \multirow{2}{*}{$\mathrm{pH}$} & \multicolumn{3}{c}{ Paramètres de Langmuir } & \multicolumn{3}{c}{ Paramètres de Freundlich } \\
\cline { 2 - 8 } & $\boldsymbol{Q}_{\mathbf{m}}\left(\mathbf{m g ~ g}^{-1}\right)$ & $\boldsymbol{K}_{\mathbf{L}}\left(\mathbf{L ~ m g}^{-1}\right)$ & $\mathbf{R}_{\mathbf{L}}$ & $\mathbf{R}^{\mathbf{2}}$ & $\boldsymbol{K}_{\mathbf{F}}{ }^{*}$ & $\mathbf{1} \mathbf{n}$ & $\mathbf{R}^{\mathbf{2}}$ \\
\hline 3 & 38,68 & 0,24 & 0,0769 & 0,9361 & 0,2042 & 0,9605 & 8,8623 \\
4 & 39,53 & 0,27 & 0,0690 & 0,9671 & 0,1622 & 0,9800 & 0,8285 \\
5 & 24,63 & 0,59 & 0,0328 & 0,8989 & 0,1479 & 1,0299 & 0,7772 \\
8 & 31,15 & 0,18 & 0,1000 & 0,9002 & 0,4266 & 0,7553 & 0,8292 \\
10 & 47,39 & 0,12 & 0,1429 & 0,8135 & 0,3311 & 0,7957 & 0,8748 \\
12 & nd & nd & Nd & 0,9980 & 0,3631 & 0,7627 & 0,9212 \\
\hline
\end{tabular}

nd : non déterminé ; *unité de $\mathrm{K}_{\mathrm{F}}:\left(\mathrm{mg} \mathrm{g}^{-1}\right)\left(\mathrm{mg} \mathrm{L}^{-1}\right)^{-1 / n}$ 


\section{DISCUSSION}

Le bioadsorbant BCM possède une large surface spécifique $\left(251,87 \mathrm{~m}^{2} \mathrm{~g}^{-1}\right)$ et présente ainsi un potentiel élevé à l'adsorption.

\section{Effet de la masse de bioadsorbant sur l'adsorption}

L'augmentation du pourcentage d'adsorption observée en fonction de la masse du bioadsorbant est due à la disponibilité de sites libres d'adsorption qui augmente avec la quantité d'adsorbant jusqu'à la masse 400 mg. Aux faibles doses de Bioadsorbant, les cations du colorant BM accèdent facilement aux sites d'adsorption du bioadsorbant, d'où l'accroissement rapide de la quantité adsorbée avec la masse du bioadsorbant. Au-delà de cette masse, le nombre de sites libres accessibles devient stable. L'addition du bioadsorbant ajoute le nombre des sites libres mais ces additions supplémentaires entraînent la formation des agglomérations des particules $\mathrm{du}$ bioadsorbant, et excluent certaines particules de bioadsorbant du processus d'adsorption; le pourcentage d'adsorption demeure ainsi constant jusqu'à $1000 \mathrm{mg}$ de bioadsorbant ajouté.

La diminution de la capacité ultime d'adsorption en fonction de la masse de Bioadsorbant s'explique par la formation des agglomérations des particules du bioadsorbant aux fortes doses en Bioadsorbant. Il y a encombrement stérique en ce moment, seules quelques particules en périphérie de la sphère formée participent réellement à l'adsorption. La capacité ultime d'adsorption exprime la quantité de soluté adsorbée par unité de masse mieux par unité des particules adsorbantes. En exprimant ainsi la capacité d'adsorption par unité de masse ou par unité particulaire, on obtient une capacité ultime d'adsorption apparente faible. L'encombrement stérique est aussi à la base de la réduction de la surface totale accessible d'où une diminution de la capacité d'adsorption en fonction de la masse, de bioadsorbant et une constance de l'adsorption à partir des masses élevées en Bioadsorbant. Il y a nivellement de l'adsorption. Vasanth et Porkodi (2007) ont aussi observé les mêmes variations de la capacité d'adsorption avec la masse de bioadsorbant pour l'adsorption de BM sur un bioadsorbant à base de Paspalum notatum.

\section{Effet du temps de contact sur l'adsorption}

L'augmentation $\mathrm{du}$ pourcentage d'adsorption ou de la capacité d'adsorption avec le temps de contact BCM-BM est due à la disponibilité des sites d'adsorption libres à la surface du bioadsorbant dès le premier contact. Ces sites se saturent progressivement. Les courbes des Figures 5, 6, 8 et 9 montrent une augmentation rapide de l'adsorption, ce qui est dû à la diffusion extraparticulaire (ou diffusion externe) des particules de l'adsorbat, caractérisée par le transfert du soluté à travers le film situé à la surface géométrique de l'adsorbant. Ensuite, on assiste à une croissance faible du pourcentage d'adsorption avec le temps, représentée par la partie tangentielle de chaque courbe. Cette étape caractérise une diffusion intraparticulaire de la matière au cours de laquelle il y a transfert des particules de l'adsorbat à travers la surface externe et la surface interne du bioadsorbant.

Le pallier observé après représente l'étape de la réaction de surface, caractérisée par la fixation des particules de l'adsorbat sur la surface interne au niveau des sites actifs des micropores, grâce aux interactions, spécifiques ou non, qui s'établissent entre les particules de l'adsorbat et les sites actifs du bioadsorbant. Le pallier indique qu'on a atteint le taux d'abattement maximal qui est de $80,75 \%$ obtenu avec les masses de $800 \mathrm{mg}$ et $1000 \mathrm{mg}$ au $\mathrm{pH}$ d'auto-équilibre 6,67 et $87,88 \%$ à pH 10 (Tableaux 2 et 3).

L'accroissement de la capacité d'adsorption en fonction du temps de contact est dû à la disponibilité des sites d'adsorption, dès que l'adsorption commence. Le pallier obtenu indique la saturation des sites d'adsorption disponibles. On atteint ainsi la capacité ultime d'adsorption $\left(Q_{\mathrm{m}}\right)$ du bioadsorbant .

\section{Cinétique d'adsorption}

Les modèles cinétiques de pseudoordre 1 et pseudo-ordre 2 ont été appliquées 
en utilisant les équations cinétiques développées par Kifuani et al. (2012). Le coefficient de corrélation global $(0,9149)$ obtenu avec le modèle de pseudo-ordre 1 est nettement plus élevé que celui obtenu $(0,8399)$ pour le modèle de pseudo-ordre 2 ; ce qui indique que le modèle cinétique de pseudo-ordre 1 est mieux approprié pour décrire l'adsorption de BM sur le bioadsorbant CM. La corrélation avec le modèle de pseudo-ordre 1 montre que l'adsorption est gouvernée par la réaction de surface caractérisée par la fixation des molécules de BM à la surface du bioadsorbant. Cette corrélation bien inférieure à 1 n'exclut pas d'autres mécanismes d'adsorption. Les courbes cinétiques ne sont pas jointes.

\section{Effet du pH sur le pourcentage d'adsorption et la capacité d'adsorption}

Le pH de la solution entraîne généralement une modification de la charge superficielle du bioadsorbant et influence aussi la dissociation du soluté. Le $\mathrm{pH}_{\mathrm{ZPC}}$ $(5,02)$ du bioadsorbant indique qu'en dessous de ce $\mathrm{pH}$, la charge de surface du bioadsorbant est positive et au-delà, la charge de surface est négative. En milieu fortement acide, très inférieur au $\mathrm{pH}_{\mathrm{ZPC}}$, les sites oxygénés de surface du Bioadsorbant sont hydrogénés et portent une charge positive. En milieu faiblement acide, il y a dissociation des sites hydrogénés du Bioadsorbant et formation des sites neutres. $\mathrm{Au} \mathrm{pH}_{\mathrm{ZPC}}$, la surface du Bioadsorbant est neutre. En milieu basique, à $\mathrm{pH} \geq \mathrm{pH}_{\mathrm{ZPC}}$, on assiste à la dissociation des fonctions hydroxylées de surface $\mathrm{du}$ Bioadsorbant et apparition des sites chargés négativement. La surface du bioadsorbant devient ainsi négative. Ces considérations sont présentées par les équations chimiques suivantes (Kifuani et al., 2012) :

$$
\begin{aligned}
& \text { A } \mathrm{pH} \leq \mathrm{pH}_{\mathrm{ZPC}}: \quad \mathrm{S}-\mathrm{OH}+\mathrm{H}_{3} \mathrm{O}^{+} \\
& \Longrightarrow \mathrm{S}_{-}-\mathrm{OH}_{2}^{+}+\mathrm{H}_{2} \mathrm{O} \\
& \mathrm{A} \mathrm{pH}=\mathrm{pH}_{\mathrm{ZPC}}: \mathrm{S}_{-} \mathrm{OH}_{2}^{+} \rightleftharpoons \mathrm{S}-\mathrm{OH}+\mathrm{H}^{+} \\
& \text {A } \mathrm{pH} \geq \mathrm{pH}_{\mathrm{ZPC}}: \mathrm{S}-\mathrm{OH}+\mathrm{OH}^{-} \\
& \longrightarrow \mathrm{S}_{-} \mathrm{O}^{-}+\mathrm{H}_{2} \mathrm{O}
\end{aligned}
$$

$\mathrm{S}$ représente la surface du bioadsorbant.
Les molécules de $\mathrm{B}^{+} \mathrm{Cl}^{-}$(ou $\mathrm{BM}$ ) sont dissociées en solution et il y a apparition des cations organiques (chargés positivement). Leur dissociation peut être représentée par l'équilibre suivant:

$$
\mathrm{BM} \rightleftharpoons \mathrm{B}^{+}+\mathrm{Cl}^{-}
$$

En présence des ions $\mathrm{B}^{+} \mathrm{du} \mathrm{BM}$ et des sites positifs du Bioadsorbant, il y a répulsion entre ces ions et ces sites chargés positivement. Il y a donc interactions répulsives entre la surface du bioadsorbant (chargée positivement) et les cations aromatiques de $\mathrm{BM}$; ce qui entraîne une diminution du pourcentage d'adsorption, en milieu fortement acide. La réduction de la capacité ultime d'adsorption en milieu acide est due aussi au phénomène de compétition $\mathrm{d}^{\prime}$ adsorption entre les cations organiques $\mathrm{B}^{+}$et les protons $\mathrm{H}_{3} \mathrm{O}^{+}$, présents dans la solution. Avec l'accroissement du $\mathrm{pH}$, il y a réduction de la répulsion des charges et donc augmentation du pourcentage d'adsorption et de la capacité d'adsorption du Bioadsorbant.

En milieu basique, il y a des interactions électrostatiques attractives entre la surface négative du bioadsorbant et les cations organiques de $\mathrm{BM}$, ce qui augmente davantage l'adsorption de BM.

Les autres interactions peuvent être des interactions attractives $\pi \quad \pi$ et $\pi$ n entre les groupements fonctionnels de surface des molécules présentes dans le bioadsorbant et les noyaux aromatiques du bleu de méthylène.

L'adsorption de BM sur le Bioadsorbant BCM est donc meilleure en milieu basique qu'en milieu acide. Le pH 10 est retenu comme $\mathrm{pH}$ optimum pour l'adsorption du bleu de méthylène sur le bioadsorbant BM. Gnana et al. (2014) ont déterminé un $\mathrm{pH}$ optimum de 8 pour l'adsorption de BM sur le bioadsorbant à base des feuilles de Syzygium cumini L. et explique aussi une meilleure adsorption de ce colorant en milieu basique par les interactions électrostatiques. Des résultats similaires ont aussi été observés par plusieurs Auteurs pour l'adsorption du bleu de méthylène ou d'autres colorants cationiques sur d'autres types d'adsorbants (Houas et al., 1999; 
Madhavakrishman et al., 2009; Luiz et al, 2011; Zhang et al ., 2011 ; Gnana et al., 2014).

\section{Isothermes d'adsorption}

Toutes les isothermes obtenues sont du type $S$ avec concavité initiale tournée vers le haut. Ce type d'isothermes traduit une adsorption multicouche (Kifuani, 2013; Masson et al., 2015). La concavité initiale tournée vers le haut indique la formation des multicouches dès les faibles concentrations du soluté en solution. Ce type d'isothermes traduit l'existence des interactions fortes entre les molécules du soluté comparées aux interactions entre molécules du soluté et l'adsorbant BCM.

Les coefficients de corrélation repris dans le Tableau 5 indiquent que le système BCM-BM peut être décrit par les modèles de Langmuir et de Freundlich ; toutefois, d'une manière générale, le modèle de Langmuir est le mieux approprié pour décrire l'adsorption de BM sur le Bioadsorbant CM (coefficients de corrélation $\mathrm{R}^{2}$ plus élevés). La corrélation avec le modèle de Langmuir suppose la formation d'une couche monomoléculaire à la surface du bioadsorbant, tous les sites d'adsorption sont équivalents et donc isoénergétiques, les interactions entre les molécules adsorbées sur la surface sont nulles ou négligeables (Laximi et Ahmazzaman, 2010 ; Nanseu-Njik et al., 2010 ; Saeed et al., 2010). La faible corrélation avec le modèle de Freundlich suppose la formation des multicouches aux concentrations élevées en bleu de méthylène (Kifuani et al., 2012).

Le paramètre $1 / n$ renseigne sur l'intensité d'adsorption, ou de la force des interactions d'adsorption. Elle indique aussi la distribution relative des sites énergétiques pour l'adsorption et dépend à la fois de la nature et de la force du processus d'adsorption (Sugana et al., 2010). Arivoli et al. (2009), Sugana et al. (2010) rapportent que si la valeur de $1 / \mathrm{n}$ est égale à 1 , l'adsorption est linéaire ; si elle est supérieure à 1 , l'adsorption est physique; si elle est inférieure à 1 , l'adsorption est un processus chimique favorable. Les valeurs de $1 / \mathrm{n}$ obtenues sont inférieures à 1 , dans la plupart des cas, et indiquent que l'adsorption de BM sur le BCM est favorable et correspond à un processus d'adsorption chimique. Ceci est en accord avec les fortes énergies d'adsorption rapportées par les paramètres $\mathrm{K}_{\mathrm{L}}$ obtenues avec le modèle de Langmuir (Tableau 5).

Le paramètre d'équilibre (ou paramètre de séparation), $\mathrm{R}_{\mathrm{L}}$, représente la nature $\mathrm{du}$ phénomène d'adsorption, qui peut être favorable $\left(0<R_{L}<1\right)$, linéaire $\left(R_{L}=1\right)$, non favorable $\left(R_{L}>1\right)$ ou irréversible $\left(R_{L}=0\right)$ (Aksu et Yener., 2001 ; Karthikeyan et Ilango, 2007 ; Kifuani et al., 2012). Les valeurs de $\mathrm{R}_{\mathrm{L}}$ (Tableau 5) obtenues comprises entre 0 et 1 indiquent que le processus d'adsorption est favorable et une chimisorption de BM sur le bioadsorbant BCM. Les capacités ultimes d'adsorption vraies calculées obtenues (Tableau 5) indiquent que le BCM est un bioadsorbant potentiel pour l'élimination du Bleu de Méthylène des eaux usées et peut donc servir pour le traitement d'épuration des eaux.

\section{Conclusion}

L'adsorption de BM sur le BCM augmente avec la masse du bioadsorbant. La concentration optimale de BCM a été estimée à $800 \mathrm{mg} / 100 \mathrm{~mL}$. Le temps d'équilibre diminue avec l'augmentation de la masse de BCM. Le temps optimum déterminé est de 150 minutes. Le taux de rabattement passe de $24,25 \%$ lorsque la masse de BCM augmente de $10 \mathrm{mg}$ à $1000 \mathrm{mg}$. Cet accroissement est attribué à la disponibilité des sites libres avec la masse de BCM. Le $\mathrm{pH}$ optimum pour l'élimination de BM par le BCM est 10 . L'adsorption de BM est meilleure en milieu basique $(87,88 \%)$ comparée au milieu acide (74,13\%); cela s'explique par le fait qu'en milieu basique, la surface du bioadsorbant est négative, d'où l'accroissement des interactions électrostatiques avec les cations organiques provenant de la dissociation des molécules de BM. L'adsorption de BM sur le BCM est mieux décrite par le modèle de Langmuir qui donne des coefficients $\mathrm{R}^{2}$ proches de 1 ; ce qui suppose une adsorption en monocouche, absence d'interactions entre les molécules adsorbées et celles encore au 
cœur de la solution. Le modèle de pseudoordre 1 décrit mieux l'adsorption de BM sur le $\mathrm{BCM}$; le mécanisme d'adsorption est donc gouverné par la réaction de surface. Les paramètres $R_{L}$ et $1 / n$ inférieurs à 1 , obtenus dans la plupart de cas, indiquent que l'adsorption de BM sur BCM est favorable. Le BCM est donc un adsorbant potentiel pour l'élimination de BM en solution aqueuse.

\section{CONTRIBUTIONS DES AUTEURS}

KKM et AKKM Sont les principaux investigateurs de cette étude et ont participé à toutes les étapes de la recherche. BIL, GEB, BMM et JML ont contribué aux traitements des données et à la discussion des résultats. BMM a aussi proposé le choix du bioadsorbant. PNV a assuré la supervision de tous les travaux.

\section{CONFLIT D'INTERETS}

Les auteurs déclarent qu'il n'y a pas de conflits d'intérêts relatifs à ce travail.

\section{REMERCIEMENTS}

$\mathrm{Au}$ moment où nous soumettons cet article pour publication, le Professeur NOKI a quitté cette terre des hommes. Nous lui exprimons ici, à titre posthume, nos vifs remerciements et nos hommages pour sa contribution à cette recherche.

\section{REFERENCES}

Aksu Z, Yener J. 2001. A comparative adsorption/biosorption study of monochlorinated phenols onto various sorbents. Waste Management, 21(8): 695-702. DOI: http//www.doi.10.1016/ SO956-053X (01)00006-X

Arivoli S, Prasath PMD, Thenkuzhali M. 2007. Adsorption of chromium ion by acid activated low cost carbon. EJEAFChe, 6(9): 2323-2340.

Arivoli S, Nandhakumar V, Saravanan S, Sulochana N. 2009. Adsorption dynamics of copper ion by low cost activated carbon. The Arabian Journal for Science and Engineering, 34(1A): 112. PMID: 40064143
Atef SA. 2009. Adsorption of methylene blue from aqueous solution onto a low-cost natural Jordan Tripoli. Amer. J. Environ. Sci., 5(3): 197-208. $\quad$ DOI: http//www.doi.10.3844/ajessp. 2

Balogoun CK, Bawa ML, Osseni S, Aina M. 2015, Préparation des charbons actifs par voie chimique à l'aide de l'acide phosphorique à base de coque de noix de coco. Int. J. Biol. Chem. Sci., 9(1): 563580 .

DOI: http://dx.doi.org/10.4314/ijbcs.v9i1.48

Berradi M, Essamri A, Al Harfi A. 2016. Discoloration of water loaded with vat dyes by membrane process of ultrafiltration. J. Mater. Sci., 7(4): 10981106.

Ertaş M, Acemioğlu B, Hakki Alma M, Usta M. 2010. Removal of methylene blue from aqueous solution using cotton stalk, cotton waste and cotton dust. J. Hazard. Mater., 183(1-3): 421-427. DOI: http//www.doi.10.1016/j.jhazmat.2010. 07.041

Gnana K, Suggala V, King P. 2014. Equilibrium and thermodynamic studies of methylene blue biosorption from aqueous solution using Syzygium cumini L. J. Environ. Res. Develop., 8(04): 964973.

Hameed BH, Ahmad AL, Latiff KNA. 2007. Adsorption of basic dye (methylene blue) onto activated carbon prepared from rattan sawdust. Dyes and Pigments, 75(1): 143-149. DOI: http//www. doi.10.1016/j.dyepig.2006.05.039

Houas A, Bakir I, Ksibi M, Elaloui. 1999. Etude de l'élimination de bleu de méthylène dans l'eau par le charbon actif commercial CECA 40. J. Chem. Phys., 96: 479-486. DOI: http//www.doi.10. 1051/jcp: 1999139

Karthikeyan G, Ilango S. 2007. Fluoride sorption using Moringa indica-based activated carbon. Iran. Environ. Health. Sci. Eng., 4(1): 21-28.

Kassale A, Barouni K, Bazzaoui M, Albourine A. 2015. Kinetics and Modeling of the adsorption of methylene blue by the 
grafted cotton. J. Chem. Bio. Phy. Sci., 5(2): 1205-1216.

Kifuani KMA, Noki VP, Ndelo DPJ, Mukana WMD, Ekoko BG, Ilinga LB, Mukinayi MJ. 2012. Adsorption de la quinine bichlorhydrate sur un charbon actif peu coûteux à base de la Bagasse de canne à sucre imprégnée de l'acide phosphorique. Int. J. Biol. Chem. Sci., 6(3): 1337-1359. DOI: http//www.doi. 10.4314/ijbcs.v6i3.36

Kifuani KMA. 2013. Adsorption des composés organiques aromatiques sur charbon actif à base des déchets agroindustriels. Thèse de Doctorat, Université de Kinshasa, 265 p.

Laximi GS, Ahmazzaman MD. 2010. Adsorption technique for the removal of phenolic compounds from wastewater using low-cost natural adsorbents. Assam University Journal of Science and Technology, 5(2): 156-166.

Li K, Zheng Z, Huang X, Zhao G, Feng J, Zhang J. 2009. Equilibrium, kinetic and thermodynamic studies on the adsorption of 2-nitroaniline onto activated carbon prepared from cotton stalk fiber. $J$. Hazard. Mater, 166(1): 213-220. DOI: http//www.doi.10.1016/j.jhazmat.2008. 11.007

Lopez-Ramon MV, Stoeckli F, MorenoCastilla C, Carrasco-Marin F. 1999. On the characterization of acidic and basic surface sites on carbons techniques. Carbon, 37: 1215-1221. DOI : http//www.doi.10.1016/S00086223(98)00317

Masson S, Reinert, Guittonneau S, Duclaux L. 2015. Cinétiques et isothermes d'adsorption de micropolluants sur un tissu et un feutre de carbone activé. Revue des Sciences de l'Eau, 28(3): 207-213. DOI : http//www.doi.10.7202/1034009ar

Madhavakrishman S, Manickavasagam K, Vasanthakumar R, Rassapan K, Mohanraj R, Pattabhi S. 2009. Adsorption of Crystal violet dye from aqueous solution using Ricinus communis pericarp carbon as adsorbent.
E-Journal of Chemistry, 6(4): 11091116. DOI: http//www.doi.10.1155/ 2009/764197

Madhura C, Priyanka S, Charmi N, Sunil K, Sonali D. 2014. Removal of dye by adsorption on various adsorbents: A review. Inter. J. Sci. Eng. And Technol. Res., 3(4):835-840.

Masson S, Reinert L, Guittonneau S, Duclaux L. 2015. Cinétique et isothermes d'adsorption de micropolluants sur un tissu et un feutre de carbone activé, Rev. Sci. de l'Eau, 28(3) : 179-247. DOI: http//www.doi.10.7202/1034009ar

Maurya MS, Mittal AK, Correl P. 2008. Evaluation of adsorption potential of adsorbents: A case of uptake of cationic dyes. J. Envir. Biol., 29(1): 31-36.

Muswema JL, Ekoko BG, Lobo KK, Ilinga L, Monama O, Mvele OM, Kifuani KM. 2010. Photodégradation de l'Acide Rouge $\mathrm{G}$ et l'Acide Orange 7 en milieu basique en présence d'une suspension de $\mathrm{TiO}_{2}$ seule ou couplée à $\mathrm{Ag}^{+}$. Rev. Cong. Sci. Nucl., 24: 1-9.

Nanseu-Njiki CP, Kenne DG, Ngameni E. 2010. Study of the removal of paraquat from aqueous solution by biosorption onto Ayous (Triplochiton schleronylon) sawdust. J. Hazard. Mater., 179(1-3): 63-71. DOI: http//www.doi.10.1016/ j.jhazmat.2010.02.058

Oliveira P, Coufort-Saudejaud C, Alliet M, Frances C. 2017. Procédé de traitement des eaux usées par boues activées : lien entre les propriétés morphologiques des flocs et l'âge de boue. Revue des Sciences de l'Eau, 30(1): 29-32. DOI: http//www.doi.10.7202/1040060ar

Saeed A, Sharif M, Iqbal M. 2010. Application potential of grapefruit peel as dye sorbent: Kinetics, equilibrium and mechanism of crystal violet adsorption. J. Hazard. Mater., 179(1-3): 564-572. DOI :

http//www.doi.10.1016/j.jhazmat.2010.0 3.041

Sakr F, Sennaoui A, Elouardi M, Tamimi M, Assabbane A. 2015. Etude de 
l'adsorption du bleu de méthylène sur un biomatériau à base de Cactus. J. Mater. Environ. Sci., 6(2): 397-406.

Sugana M, Siva Kumar N, Venkata Subbaiah M, Krishnaiah. 2010. Removal of divalent manganese from aqueous solution using Tamarindus indica fruit nut Shell. J. Hazard. Mater., 2(1): 7-20.

Sun L, Yu H, Fugetsu B. 2012. Graphene oxide adsorption enhanced by in situ reduction with sodium hydrosulfite to remove Acridine orange from aqueous solution. J. Hazard. Mater., 203-204: 101-110. DOI: http//www.doi. 10.1016/j.jhazmat.2011.11.097

Tchuifon TDR, Nche G, Tchouanyo DH J, Ngakou SC, Djoumbissie AL, Anagho SG, Kamgaing T, Ketcha JM. 2015. Adsorption studies of methylene blue on methanol modified and unmodified corn stalks and egussi peeling, Inter. J. Engin. and Techn. Res.(IJETR), 3: 8-14.

Vanessa P, Andrin A, Le Bechec M, Lacombe S, Frayret J, Pigot T. 2017. Couplage photocatalyse-oxydation par le ferrate (VI) pour le traitement du colorant rhodamine 6G. Revue des Sciences de l'Eau, 30(1): 35-39. DOI: http//www.doi.10.7202/1040061ar

Vasanth K, Porkodi K. 2007. Mass transfer, kinetics and equilibrium studies for the biosorption of Methylene blue using Paspalum notatum. J. Hazard. Mater., 146(2): 214-226. DOI: http//www.doi.10.1016/j.jhazmat.2006.1 2.010

Yao MK, Kouassi NL-K, 2015, Etude des propriétés d'adsorption et de désorption $\mathrm{du} \mathrm{Pb}$ et $\mathrm{Cd}$ par les sédiments d'une lagune tropicale en présence d'Allythiourée. Int. J. Biol. Chem. Sci., 9(1): $\quad 483-491 . \quad$ DOI: http//www.doi.10.4314/ijbcs.v9i1.41

Zhang L, Xu T, Liu X, Zhang Y, Jin H. 2011. Adsorption behavior of multi-walled carbon nanotubes for the removal of olaquindox from aqueous solutions. $J$. Hazard. Mater, 197: 389-396. DOI: http//www.doi.10.1016/j.jhazmat.2011.0 9.100

Zidane F, Ohazzar A, Blais JF, Ayoubi K, Bensaid J, Basri SE, Kaba N, Fakhreddine Q, Lekheif B. 2011. Contribution à la dépollution des eaux usées de textile par électrocoagulation et par adsorption sur des composés à base du fer et d'aluminium. Int. J. Biol. Chem. Sci., 9(1): 1727-1745. DOI: http//www.doi.10.4314/ijbcs.v5i4.35 